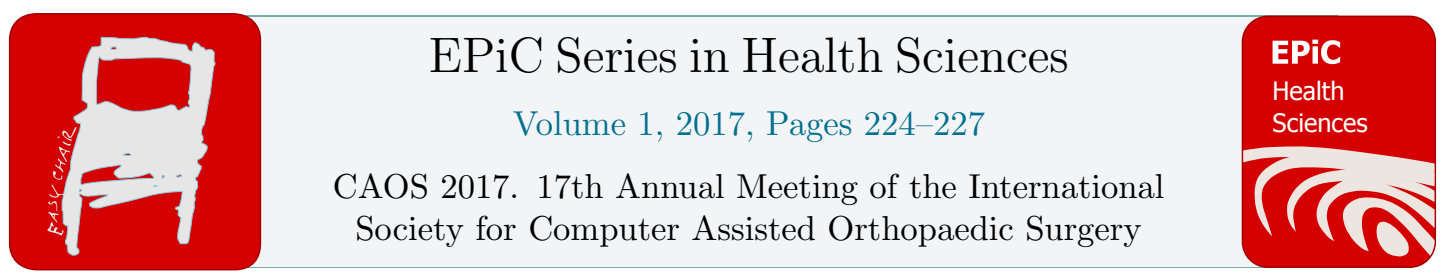

\title{
Quantifying Dynamic Assessment of Developmental Dysplasia of the Hip
}

\author{
Olivia Paserin BEng ${ }^{1 *}$, Niamul Quader MSc ${ }^{1}$, Kishore Mulpuri MBBS ${ }^{2}$, \\ Anthony Cooper $\mathrm{MBChB}^{2}$, Emily Schaeffer $\mathrm{PhD}^{2}$, Antony J Hodgson $\mathrm{PhD}^{3}$, \\ and Rafeef Abugharbieh $\mathrm{PhD}^{1}$ \\ 1,* Department of Electrical and Computer Engineering, University of British Columbia, \\ Vancouver, Canada \\ ${ }^{2}$ Department of Orthopedic Surgery, British Columbia's Children's Hospital, Vancouver, Canada \\ ${ }^{3}$ Department of Mechanical Engineering, University of British Columbia, Vancouver, Canada \\ opaserin@ece.ubc.ca
}

\section{Introduction}

Although physical and ultrasound-based screening for congenital deformities of the hip (developmental dysplasia of the hip, or DDH) is routinely performed in most countries, one of the most commonly performed maneuvers done under ultrasound observation - dynamic assessment (Harcke 1990, CPG 2000) - has been shown to be relatively unreliable and is associated with significant misdiagnosis rates, on the order of 29\% (Imrie 2010).

In a dynamic assessment of an infant's hip, clinicians apply stress to the adducted hip, in a posterior direction to provoke dislocation, and observe the resulting joint movement with ultrasound (US) (Harcke 1990). The resulting observations are currently described qualitatively using terms such as normal, lax, dislocatable, reducible and not reducible, which are not based on measured quantities. Inter-assessor variability likely accounts for the poor reproducibility of dynamic assessments and the observed rates of misdiagnosis. Our overall research objective is therefore to develop a quantitative method of assessing hip instability in order to improve reliability of diagnosis and reduce misdiagnosis rates.

Our group has recently developed an approach to automatically calculate femoral head coverage (FHC) in 2D US scans, a ratio describing how much of the femoral head is sitting in the acetabular cup of the hip joint (publication conditionally accepted). While the inter-rater variability of manual FHC measurements in static assessments has been found to be about 9\% (Peterlein 2010), it is not yet known what typical changes in FHC during dynamic assessments are since previous approaches are based on qualitative descriptors only. The purpose of this study is thus to evaluate the feasibility of applying our recently-developed automatic FHC assessment process to estimate the change in FHC during a dynamic assessment and to compare this change with the known variability of the measurement (prior to designing a clinical study). 


\section{Materials and Methods}

The FHC ratio is illustrated in Figure 1; FHC is calculated as the ratio of the distance between the planar portion of the ilium closest to the hip joint and the point of the femoral head deepest in the acetabulum (d) to the femoral head's diameter (D). With stress applied to a stable hip joint, we expect FHC to vary minimally. On the other hand, an unstable hip would express large changes in FHC as the femoral head does not sit well in the acetabular socket. Thus, we use $\triangle \mathrm{FHC}$ to quantify joint stability, where $\triangle \mathrm{FHC}=\mathrm{FHC}_{\max }-\mathrm{FHC}_{\min }$.

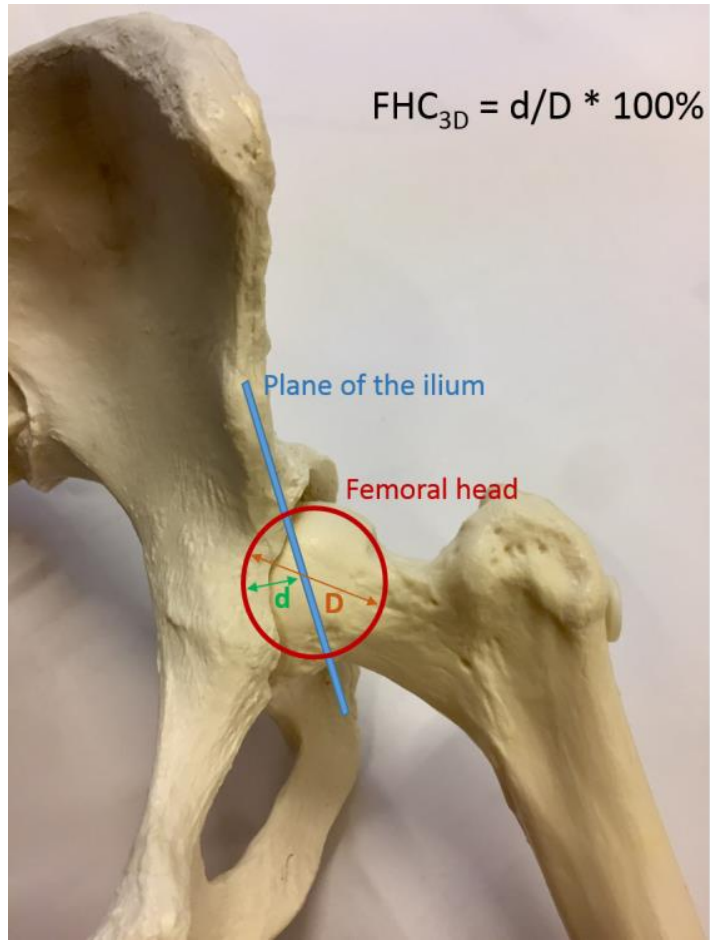

(a)

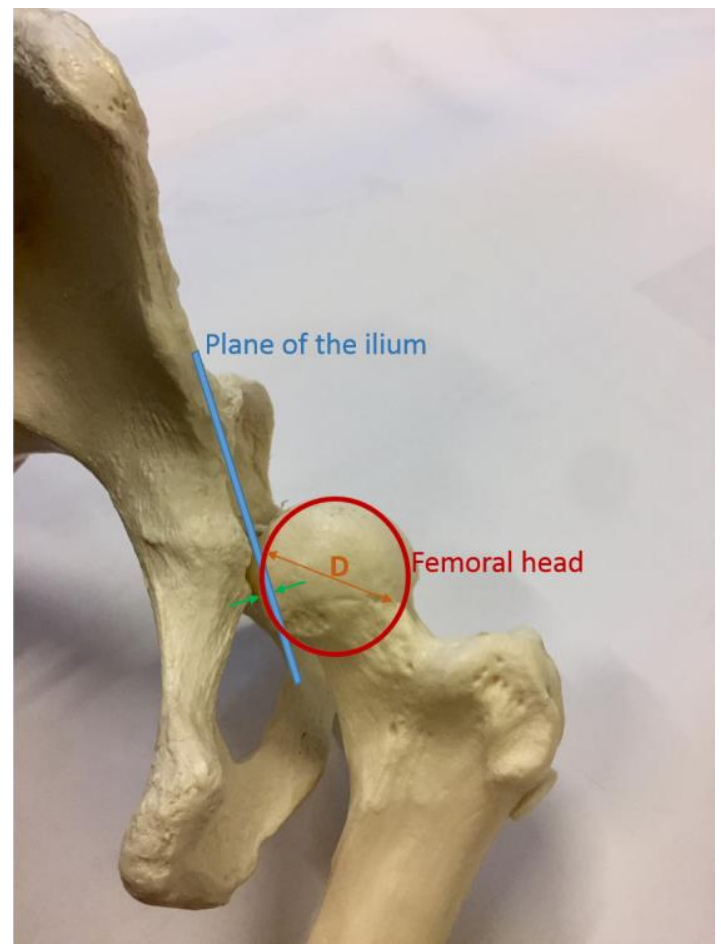

(b)

Figure 1: Demonstrating how femoral head coverage relates to the anatomy of the hip joint since it corresponds to the degree that the femoral head sits inside the acetabulum. Large variations in FHC, as from (a) to (b), indicate dynamically unstable hips.

For this pilot study, an attending pediatric orthopedic surgeon performed one dynamic assessment on an infant and acquired five B-mode 3D US volumes from the left hip of the infant with varying levels of distraction force applied (obtained as part of routine clinical care under appropriate institutional review board approval using a SonixTouch Q+ machine with a 4DL14-5/38 linear 4D transducer set at $7 \mathrm{MHz}$ ). To automatically estimate FHC in each of the US volumes, we segmented the planar portion of the ilium nearest the hip joint using phase-symmetry responses (Quader 2016) and segmented the femoral head by uniformly sampling each US volume into 2D US slices and creating a pixel-wise probability map of finding the femoral head at each voxel of the US volume. Once both the ilium and femoral head were segmented, FHC was computed as FHC $=\mathrm{d} / \mathrm{D} * 100 \%$. Finally, we estimated the joint laxity by computing $\triangle \mathrm{FHC}=\mathrm{FHC}_{\max }-\mathrm{FHC}_{\min }$ among the set of five US volumes. 


\section{Results}

Segmentation accuracy: We compared our femoral head segmentation results against manually labelled spherical femoral heads in all five US volumes. We found significant overlaps between the automatic and manual segmentations - Jaccard of 0.86 (standard deviation 0.04) and Dice coefficient of 0.93 (standard deviation 0.02).

Quantitative $\triangle \mathrm{FHC}$ vs. qualitative assessment: We found a $\triangle \mathrm{FHC}$ of $19 \%$ in the infant hip we examined $(55 \%-74 \%)$. Our clinician's qualitative assessment concluded the hip to be normal and not indicative of instability.

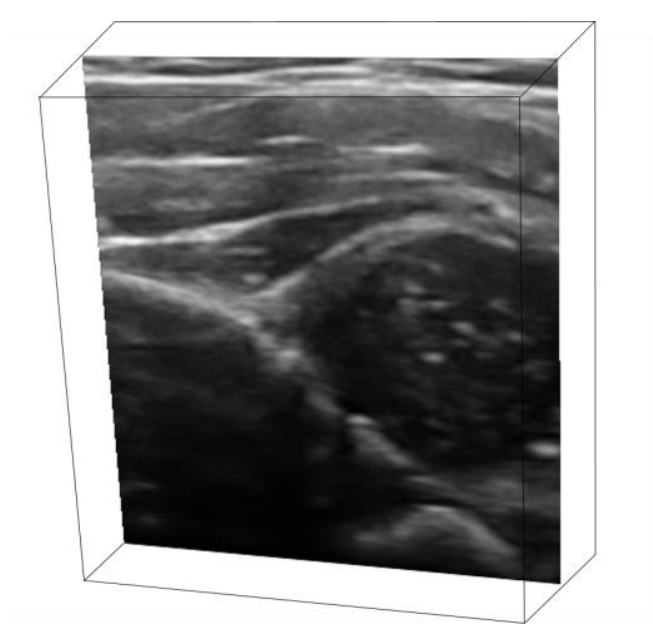

(a)

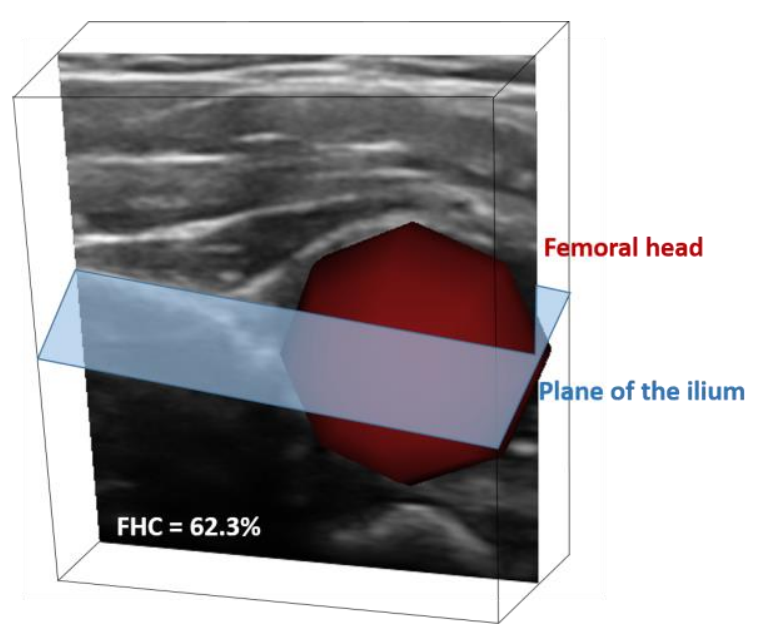

(b)

Figure 2: (a) 3D visualization of the raw ultrasound data with one coronal slice from the volume displayed. In (b), the segmentation of the plane extending from the ilium toward the hip joint and the segmentation of the spherical femoral head are shown.

\section{Discussion}

In this study, we were able to apply our recently-developed automatic FHC assessment process to estimate the change in FHC during a dynamic assessment and found that the femoral centre moved by up to $19 \%$ of its diameter during distraction. Since the inter-rater, inter-exam variability in measuring FHC is approximately $9 \%$ (Peterlein 2010) (with typical FHC values $>50 \%$ in normal hips and $<50 \%$ in dysplastic hips (Bond, 1997, Gunay 2009)), a value of $19 \%$ for $\triangle \mathrm{FHC}$ would indicate that the distraction force likely produced a real lateral displacement. Nonetheless, the attending clinician felt that this degree of distraction was relatively normal and not indicative of instability. This suggests that our technique likely has sufficient resolution and repeatability to quantify differences in laxity between stable and unstable hips, although this presumption will have to be confirmed in a subsequent study with additional subjects. In future work, we plan to collect more US-recorded dynamic assessments in order to determine a reliable range of $\triangle \mathrm{FHC}$ for both stable and dysplastic infant hips. The long-term significance of this approach to evaluating dynamic assessments may lie in increasing early diagnostic sensitivity in order to prevent dysplasia remaining undetected prior to manifesting itself in early adulthood joint disease. 


\section{Disclosures}

No relevant disclosures.

\section{References}

Bond CD, Hennrikus WL, DellaMaggiore ED, Prospective evaluation of newborn soft-tissue hip "clicks" with ultrasound, Journal of Pediatric Orthopaedics, 17(2), pp: 199-201, 1997. Voronkov, A. (2004). EasyChair conference system. Retrieved from easychair.org

Clinical Practice Guideline (CPG): Committee on Quality Improvement, \& Subcommittee on Developmental Dysplasia of the Hip, Early detection of developmental dysplasia of the hip, Pediatrics, 105(4), 896, 2000. Wikipedia. (n.d.). EasyChair. Retrieved from Wikipedia: https://en.wikipedia.org/wiki/EasyChair

Gunay C, Atalar H, Dogruel H, Yavuz OY, Uras I, Sayh U, Correlation of femoral head coverage and Graf alpha angle in infants being screened for developmental dysplasia of the hip, International Orthopaedics, 33, pp: 761-764, 2009. Voronkov, A. (2014). Keynote talk: EasyChair. In Proceeedings of the 29th ACM/IEEE International Conference on Automated Software Engineering (pp. 3-4). ACM.

Harcke HT, Grissom LE, Performing dynamic sonography of the infant hip, American Journal of Roentgenology, 155(4), pp: 837-844, 1990.

Imrie M, Scott V, Stearns P, Bastrom T, Mubarak SJ, Is ultrasound screening for DDH in babies born breech sufficient, Journal of Children's Orthopaedics, 4(1), pp: 3-8, 2010.

Peterlein CD, Schuttler KF, Lakemeier S, Timmesfeld N, Gorg C, FuchsWinkelmann S, Schofer MD, Reproducibility of different screening classifications in ultrasonography of the newborn hip, BMC Pediatrics, 10, pp: 98, 2010.

Quader N, Hodgson A, Mulpuri K, Cooper A, Abugharbieh R, Towards Reliable Automatic Characterization of the Neonatal Hip Dysplasia from 3D Ultrasound Images, MICCAI, 2016. 